\title{
CLASSIFICAÇÃO DE VEÍCULOS HÍBRIDOS
}

\section{- EVOLUÇÃO CRESCENTE DO GRAU DE ELECTRIFICAÇÃO}

\section{Resumo}

Em resposta aos impactos ambientais e limitações dos combustíveis fósseis, assistiu-se nas últimas décadas a um acentuado desenvolvimento dos veículos híbridos elétricos convencionais. A sua proliferação encontra-se hoje bem disseminada, em praticamente todas as gamas, refletindo a confiança dos consumidores. Com vista a atenuar ainda mais o uso dos combustíveis fósseis, a tendência é de aumentar o nível de eletrificação nas versões híbridas mais recentes, bem como da oferta de versões puramente elétricas. No entanto, a evolução dos últimos anos, quer ao nível da aposta por parte dos fabricantes, quer ao nível do volume de vendas, parece indiciar uma nova fase de proliferação destes veículos, a qual se encontra ainda a dar os primeiros passos.

Este artigo apresenta uma classificação dos veículos híbridos, em função do nível de eletrificação do sistema de propulsão. Após uma breve referência aos veículos puramente elétricos, fazem-se algumas considerações acerca do impacto mundial dos veículos híbridos Plug-in e puramente elétricos, nos últimos 5 anos.

\section{Introdução}

As limitações das reservas de combustíveis fósseis e flutuações de preços, bem como o impacto ambiental das emissões de gases de efeito de estufa, têm motivado a procura de soluções energéticas alternativas, por parte de governos e fabricantes de automóveis. Com efeito, é no sector dos transportes que tais alternativas são mais urgentes: nos países membros da OCDE, 60\% do consumo de produtos petrolíferos deve-se a este sector, sendo responsável por cerca de $25 \%$ das emissões globais de $\mathrm{CO} 2$ [1]. De acordo com esta referência, os transportes rodoviários estão associados à maior fatia - $75 \%$ do consumo total neste sector.

Nos últimos 20 anos, os veículos híbridos elétricos (VH) e puramente elétricos (VE), têm vindo a assumir-se como as alternativas mais promissoras aos veículos convencionais, baseados no motor de combustão interna (MCl). Para tal, têm contribuído os avanços em múltiplos domínios tecnológicos, tais como as máquinas elétricas, eletrónica de potência, sistemas de controlo e de armazenamento de energia [2], [3].

Embora sejam de registar avanços significativos nas tecnologias das baterias, há limitações importantes que não foram ainda superadas (e.g., preço, peso e volume elevados, densidades de potência e autonomias baixas, com tempos longos de carregamento). Sendo uma tecnologia considerada promissora, as células de combustível encontram-se ainda numa fase de desenvolvimento incipiente [3].

Estas desvantagens são responsáveis pela reduzida disseminação dos VE. Por seu turno, os VH aliam as características dos veículos convencionais às vantagens da propulsão elétrica (rendimentos mais elevados com menores emissões de gases poluentes e recuperação da energia de frenagem), sem as limitações de autonomia dos VE. Naturalmente, isto explica a maior aceitação verificada por parte dos consumidores. Dependendo do grau de eletrificação do sistema de propulsão, os preços dos VH podem ser bastante mais elevados do que as opções convencionais $(\mathrm{MCl})[3]$.

As pressões crescentes de governos locais e regionais, quanto às limitações de emissões poluentes (em particular nos centros urbanos), têm motivado a indústria automóvel a intensificar e diversificar os seus esforços: por um lado, a melhoria contínua dos desempenhos, fiabilidade, segurança e conforto dos veículos convencionais, com um apertado controlo de custos [3], [4]; por outro, uma clara aposta no aumento da oferta comercial de VH e VE, bem visível nos 
últimos 5 anos. No caso dos $\mathrm{VH}$, a tendência é para uma crescente eletrificação do sistema de propulsão. Disso são exemplo os veículos Plug-in (PHEV) e Range Extended Electric Vehicle (REEV).

O principal objetivo deste artigo é apresentar uma classificação dos $\mathrm{VH}$, associada à eletrificação da propulsão do veículo.

Far-se-á também uma breve referência ao impacto que as versões mais eletrificadas têm tido nos últimos anos. Deste modo, a estrutura do artigo é a seguinte: A secção 2 apresenta uma classificação dos VH baseada no índice de eletrificação do sistema de propulsão, de acordo com a terminologia usada pelos fabricantes. Segue-se uma breve descrição dos tipos de VE. A secção 3 faz uma análise sucinta da evolução das vendas de PHEV e VE (baterias) - global e local -, nos últimos 5 anos. Na secção 4 apresentam-se as conclusões.

\section{Classificação com base no nível de eletrificação}

A classificação apresentada nesta secção está intimamente relacionada com as características do sistema de propulsão considerado. Num artigo publicado em edição anterior [5], foram apresentados os principais tipos de propulsão atualmente utilizados nos VH e VE. Sempre que necessário, farse-á aqui referência a essas configurações. A Figura 1 apresenta as designações mais usuais, associadas à eletrificação do sistema de propulsão.

$\mathrm{Na}$ literatura sobre este assunto, as definições de VH ou VE nem sempre são totalmente coincidentes. No presente artigo, consideram-se $\mathrm{VH}$ aqueles onde esteja presente o $\mathrm{MCl}$; os sistemas de propulsão que integrem apenas máquinas elétricas são aqui designados como VE. Como é visível na figura, há algumas diferenças em relação às definições usadas em [6].

\subsection{Veículos Híbridos}

Neste grupo estão incluídas as designações HEV (híbridos convencionais), PHEV e REEV (Figura 1).

Na primeira, o papel da componente elétrica é secundário, sendo muito mais relevante nos veículos PHEV e REEV (nestes últimos a propulsão elétrica é mesmo a única).

A sequência das descrições seguintes assenta num grau crescente do nível da componente elétrica na propulsão do veículo: tal implica uma diminuição da potência do $\mathrm{MCl}$, acompanhada do aumento da potência do(s) motor(es) elétrico(s) (ME), bem como do(s) conversor(es) de potência e das baterias. Tal contribui para um aumento do preço final e peso do veículo, em relação às versões $\mathrm{MCl}$.

Como referido na introdução, o estado atual da tecnologia das baterias tem aqui uma influência muito significativa.

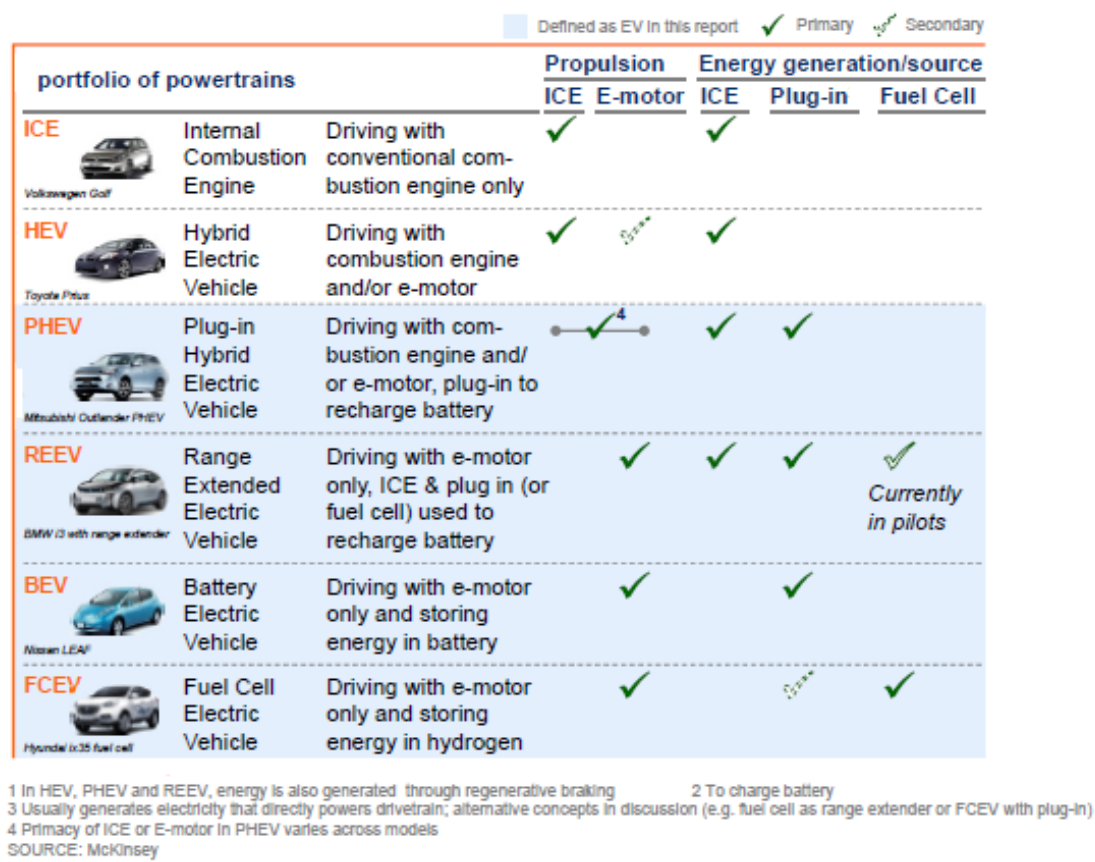
sPUinacy of ICE or E-motor In PHEV varles across models

Figura 1. Designações dos Sistemas de Propulsão (função do nível de eletrificação) [6] 


\subsubsection{Híbridos Convencionais [7], [8]}

Neste grupo, são usualmente considerados três níveis, em função da integração elétrica: micro, mild e full hybrid.

\section{- Micro hybrid}

Face aos veículos convencionais, a diferença está na possibilidade do gerador incorporado poder funcionar como motor, durante o arranque do $\mathrm{MCl}$ (Integrated Starter Generator - ISG). Deste modo, conseguem-se poupanças de energia quando o veículo está parado ( $\mathrm{MCl}$ desligado). Algumas variantes com geradores de maior potência permitem frenagens regenerativas (em alguns documentos são designadas como Micro-Mild hybrid). Claramente vocacionado para condução citadina (arranques e paragens frequentes). O seu custo é ligeiramente superior ao veículo convencional, dado o reduzido grau de eletrificação.

\section{- Full hybrid}

O sistema de propulsão é do tipo série - paralelo ou série paralelo complexa (ver [5]), havendo duas máquinas elétricas (motor e gerador) e $\mathrm{MCl}$. Os sistemas de transmissão de potência são mais complexos (e.g. engrenagens planetárias), tornando mais flexível a divisão da potência requerida (entre $\mathrm{MCl}, \mathrm{ME}, \mathrm{GE}$ e sistema de armazenamento de energia). Deste modo, o desempenho do $\mathrm{MCl}$ é otimizado (i.e., rendimento máximo, com emissões mínimas). São possíveis os seguintes modos de propulsão (incluindo frenagens regenerativas):

i. $\mathrm{ME}$ (arranque/paragem);

ii. $\mathrm{MCl}$ (modo "cruising", desempenho ótimo);

iii. Modo combinado: $\mathrm{ME}$ (ou GE) + $\mathrm{MCl}$. Por exemplo, o ME pode auxiliar o $\mathrm{MCl}$ nas acelerações; no caso da potência requerida ser inferior ao "ótimo ICE", o excedente é convertido em energia elétrica pelo $\mathrm{GE}$, sendo armazenada nas baterias.

\subsubsection{PHEV (Plug-in Vehicles) [7], [8]}

O sistema de propulsão é semelhante ao "Full-Hybrid". As baterias são carregadas através de uma fonte exterior de energia elétrica, aproveitando também as frenagens regenerativas. O sistema de baterias tem maior capacidade, comparando com as configurações anteriores, embora menor do que nos veículos puramente elétricos (VE). São de destacar os seguintes modos de propulsão:

i. Trajetos curtos - Apenas propulsão elétrica, pelo que são as baterias que fornecem a energia requerida;

ii. Trajetos longos - Quando o nível de carga das baterias (SOC) é inferior a um valor fixado, a propulsão $\mathrm{MCl}$ é ativada (modo híbrido).

A Figura 2 sintetiza as principais características das variantes descritas.

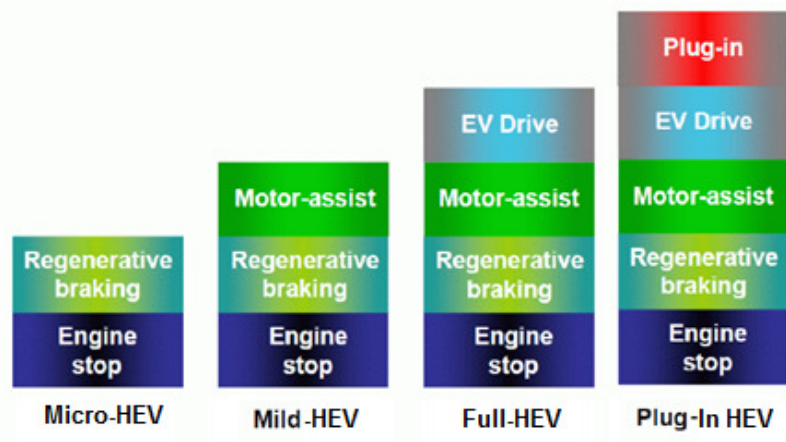

Figura 2. Sistemas de Propulsão: Híbridos Convencionais (micro, mild e full) e PHEV

É de realçar que a propulsão puramente elétrica apenas existe em Full-HEV e PHEV. Em todas estas variantes, o $\mathrm{MCl}$ participa diretamente no esforço de tração; tal não sucede na configuração seguinte (mais recente), na linha de uma maior relevância da componente elétrica.

\subsubsection{REEV (Range Extended Electric Vehicles) [6]}

Em termos de topologia, estes veículos assentam no sistema de propulsão série (ver [5]). Semelhantes aos PHEV (incluindo a possibilidade do carregamento exterior das baterias), a principal diferença dos REEV está no facto da propulsão ser feita exclusivamente por motores elétricos.

Como tal, o $\mathrm{MCl}$ funciona como máquina primária do gerador, sendo responsável pelo carregamento das baterias, somente quando a respetiva carga atinge um valor mínimo especificado. 
Deste modo, o $\mathrm{MCl}$ permite estender a autonomia do veículo, em relação à carga inicial das baterias, proveniente da fonte exterior.

O peso e volume das baterias são normalmente menores, em comparação com a variante puramente elétrica (dependente da potência do gerador e da capacidade do depósito de combustível). Trata-se de um conceito recente, muito próximo dos veículos puramente elétricos. Esta configuração permite contornar o risco de a bateria ficar sem carga, durante o percurso previsto. Com efeito, este receio tem uma enorme influência no modo como os veículos puramente elétricos são encarados pela generalidade dos utilizadores.

\subsection{Veículos elétricos (VE) [5]}

Apenas incluem motores elétricos. Em termos de fontes de energia empregues há a distinguir as baterias das células de combustível.

\subsubsection{Baterias}

Atualmente, as baterias mais usadas nos VE e VH são as de hidretos metálicos de níquel (NiMH) e de iões de lítio (LiIon). Particularmente nestas últimas, têm sido obtidos aumentos consideráveis nos valores da densidade de energia (de momento apresentam valores muito superiores aos restantes tipos de baterias). O tamanho e volume das baterias condiciona o projeto da estrutura mecânica do veículo, bem como o seu preço. Há uma clara tendência para a sua integração com supercondensados, aproveitando os elevados valores de densidade de potência destes últimos

\subsubsection{Células de Combustível}

Embora apresentem um potencial elevado como alternativa aos veículos convencionais, esta tecnologia está ainda numa fase inicial. Trata-se de geradores de energia elétrica, resultante de reações eletroquímicas entre hidrogénio (combustível não poluente, com elevada densidade de energia, mas reduzida densidade de potência) e oxigénio. 0 produto destas reações é apenas vapor de água.
São, pois, dispositivos distintos das baterias (armazenadores de energia). A energia elétrica produzida nas células de combustível é usada na propulsão do veículo ou fornecida ao sistema de armazenamento de energia do veículo (e.g., baterias e supercondensados). O seu custo e, sobretudo, a necessidade de infraestruturas de fornecimento de hidrogénio colocam sérias interrogações sobre a sua proliferação.

Com base nas designações anteriores, a Tabela 1 apresenta alguns valores de referência sobre poupança de energia (em relação a veículos convencionais $\mathrm{MCl}$ ), potência nominal do motor elétrico e níveis de tensão do sistema elétrico do veículo.

Tabela 1. Especificações por tipo de veículo [7], [8]

\begin{tabular}{|c|c|c|c|c|c|}
\hline Functions & $\begin{array}{c}\text { Micro } \\
\text { Hybrid }\end{array}$ & $\begin{array}{c}\text { Mild } \\
\text { Hybrid }\end{array}$ & $\begin{array}{c}\text { Full } \\
\text { Hybrid }\end{array}$ & $\begin{array}{l}\text { Plug in } \\
\text { hybrid } \\
\text { (PHEV) }\end{array}$ & EV \\
\hline $\begin{array}{l}\text { Energy } \\
\text { Savings }\end{array}$ & $\begin{array}{c}5-10 \% \\
\text { (upto } 25 \% \\
\text { in city } \\
\text { traffic) }\end{array}$ & $\begin{array}{c}10- \\
25 \%\end{array}$ & $25-40 \%$ & $50-100 \%$ & $100 \%$ \\
\hline $\begin{array}{c}\text { Electric Motor } \\
\text { or ISG (1) } \\
\text { (kW) }\end{array}$ & $3-5$ & $7-12$ & 40 & $30-70$ & $50-100$ \\
\hline $\begin{array}{l}\text { System (1) } \\
\text { Voltage } \\
\text { (V) }\end{array}$ & $12-42$ & $150-200$ & $200-350$ & $300-500$ & $300-500$ \\
\hline
\end{tabular}

(1) Massa do veículo inferior a $2000 \mathrm{~kg}$

Na Figura 3 está ilustrada uma síntese desta secção, sendo de destacar as estimativas de redução de emissões de $\mathrm{CO} 2$ (em relação a veículo equivalente $\mathrm{MCl}$ ) e o custo do veículo.

\section{Powertrain Electrification - A Technical Evolution}

\section{Fuel Efficiency Improvement} Fuel Substitution

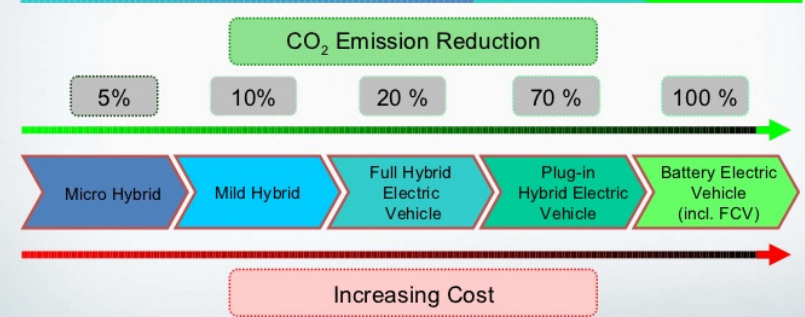

Figura 3. Sistemas de Propulsão: impacto da eletrificação [9] 
No caso dos PHEV, BEV FCV é importante sublinhar que se tratam somente de reduções locais de emissões. O impacto ambiental real deverá contabilizar também as emissões associadas à produção da energia elétrica fornecida ao veículo (PHEV e BEV), bem como na produção de hidrogénio (FCV).

\section{Impacto dos Veículos ${ }^{1}$ Híbridos e Elétricos}

A evolução das alternativas aos veículos convencionais $(\mathrm{MCl})$, por parte da indústria automóvel, coincide com a classificação apresentada na secção anterior. O lançamento do Toyota Prius, em 1997, marcou o início de tais alternativas. Este e outros modelos híbridos convencionais (HEV), de múltiplos fabricantes, são atualmente comercializados em todo o mundo, apresentando bons desempenhos dinâmicos e níveis de consumo [5]. Na Europa, América do Norte e em alguns países asiáticos (China e Japão), estas soluções alcançaram um nível significativo de implantação no mercado automóvel. A título de exemplo, veja-se o Toyota Prius: de acordo com [6], desde o início da sua comercialização foram vendidos mais de 6 milhões de unidades.

O cenário relativo aos veículos com maior grau de eletrificação (PHEV, REEV, e elétricos puros - baterias (VE-B) e células de combustível (VE-FC)) é bem diferente, com um impacto ainda muito reduzido (em termos globais, menos de 1\% do registo de veículos novos, em 2015 [10]). No entanto, nos últimos 4 anos o seu crescimento tem sido exponencial ${ }^{2}$. Em 2015, mais de 1 milhão e 200 mil PHEV e VE-B circulavam em todo o mundo (Figura 4). Tal evolução parece significar o início de uma nova fase de aceitação e proliferação destes veículos.

No entanto, há diversas incertezas quanto à sua proliferação, resultantes do processo de interação entre fabricantes (características tecnológicas e custos), consumidores (aceitação) e governos (medidas de incentivo à sua adoção). O que é seguro afirmar é que estes resultados são fruto dos esforços concertados que têm vindo a ser realizados pela indústria automóvel e órgãos decisores.

Em seguida, realçam-se apenas algumas tendências sobre a aceitação de PHEV e VE-B à escala mundial. A Figura 4 representa o crescimento do número de PHEV+ VE-B, nos últimos 6 anos.

A Figura 5 representa o volume de vendas de PHEV + VE-B, desde 2010 até 2015 . As respetivas quotas de mercado em 2015 estão também incluídas.

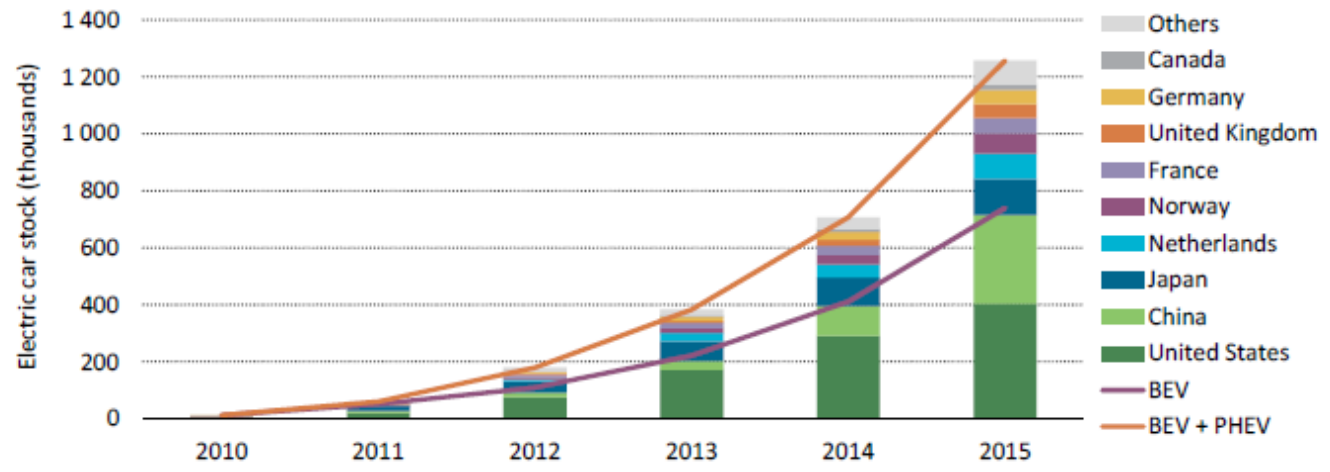

Figura 4. Evolução do número de PHEV+ VE-B [10]

(1) Refere-se apenas a ligeiros.

(2) Alguns exemplos: o Nissan Leaf, lançado em 2011, atingiu as 100000 unidades vendidas em 2013. Do modelo S da Tesla, lançado nesse ano, foram já vendidas 25000 unidades [6]. 


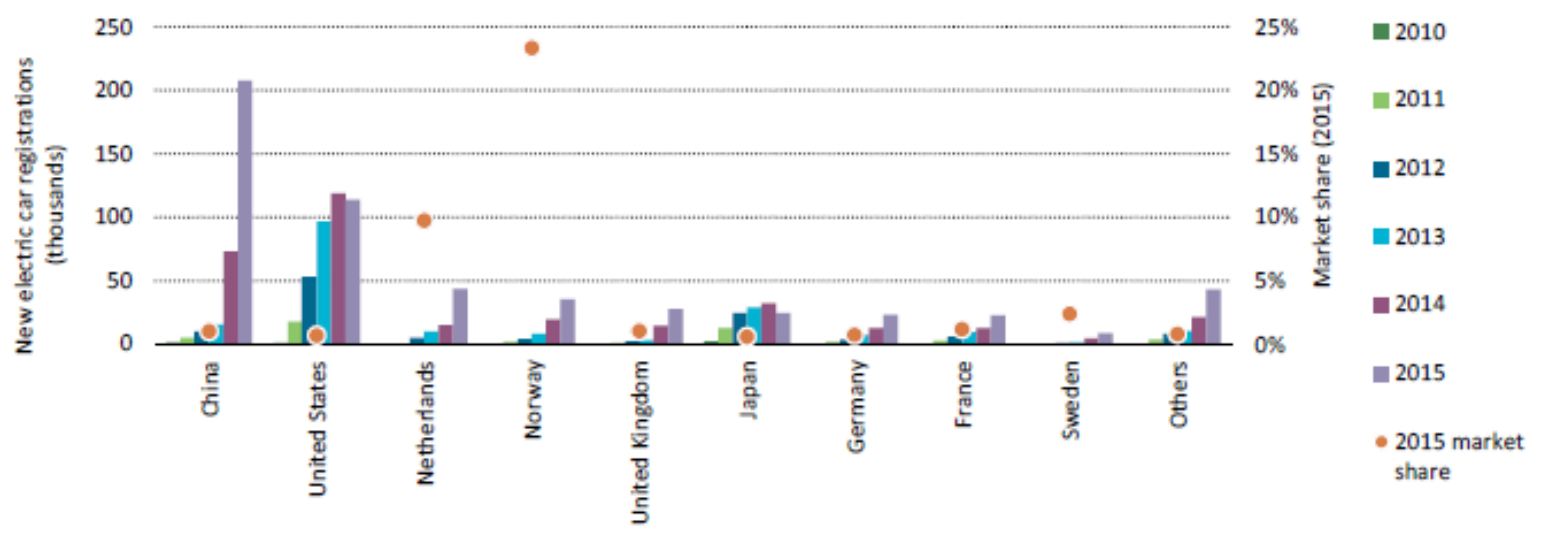

Figura 5. Vendas de PHEV+ VE-B (2010-2015) e quotas de mercado (2015) [10]

A partir de 2011, assistiu-se a um aumento substancial da proliferação destes veículos (notar que a quota dos países indicados na Figura 5 corresponde a $90 \%$ do total).

A maior recetividade verifica-se nos países com maior desenvolvimento económico e industrial (ou em vias de...), sendo os EUA líderes neste ranking, seguidos da China, Japão e Europa (Figura 4).

Em 2015 há a registar um aumento global muito significativo de PHEV e VE-B (cerca de 70\% em relação a 2014), mas desigual, consoante a região do globo. Na China, o número destes veículos aproximou-se muito do valor dos EUA: o elevado aumento das vendas chinesas (cerca de 2,7 vezes o valor de 2014), juntamente com uma ligeira diminuição das vendas americanas, explicam aquela aproximação (Figura 5). Com efeito, as quotas conjuntas de PHEV e VE-B nestes dois países ultrapassaram os $50 \%$ em 2015. Curiosamente, é somente nos EUA e Japão (países onde a aceitação daqueles veículos começou por ser maior) que as vendas diminuem (em 2015 e pela primeira vez). De facto, até 2014 o somatório das quotas destes dois países era superior a $50 \%$ da totalidade dos veículos registados. Em 2015, tal somatório correspondeu a cerca de 40\% (Figura 4).

No caso Europeu, a quota destes veículos tem-se mantido constante, em cerca de $1 / 3$ do valor total. De referir que no último ano, as vendas de PHEV e VE-B na Holanda e Noruega atingiram percentagens muito significativas nos respetivos mercados: $10 \%$ no primeiro caso e $23 \%$ no segundo (Figura $5)$.
Tais valores demarcam-se claramente dos restantes e são um bom exemplo da importância de medidas eficazes que estimulem os consumidores a optar por soluções elétricas de mobilidade.

Este e outros assuntos (e.g., nichos com maior potencial de aplicação PHEV e VE-B, gamas de veículos, números de PHEV e VE-B) justificam um tratamento em espaço próprio; num próximo artigo, procurar-se-á fazer uma abordagem com maior profundidade.

\section{Conclusões}

O sector dos transportes depende ainda em grande escala dos combustíveis fósseis. As limitações das reservas disponíveis e o impacto ambiental, têm motivado a procura e desenvolvimento de soluções alternativas, sobretudo nos transportes rodoviários. Atualmente, os VH e VE constituem a alternativa mais viável aos veículos convencionais ( $\mathrm{MCl})$, não obstante as dificuldades técnicas e incertezas quanto à sua aceitação, que ainda subsistem.

Neste artigo apresentou-se uma classificação dos VH, de acordo com o nível de eletrificação do sistema de propulsão do veículo. Os últimos 20 anos ficaram marcados pela chegada e permanência dos veículos híbridos elétricos, no sector automóvel. A tecnologia híbrida convencional tem hoje uma implantação sólida, com um vasto leque de oferta, por parte dos principais fabricantes de automóveis. Desde 2010 tem-se verificado uma tendência de aumento do grau de eletrificação dos sistemas de propulsão, materializada nos PHEV e VE-B. 
Os desenvolvimentos destas tecnologias, incentivados pela legislação ambiental e incentivos à compra destes veículos parecem estar a dar frutos, no que se refere à sua aceitação e confiança.

\section{Referências}

[1] "Transport, Energy and CO2 - Moving Toward Sustainability", International Energy Agency, 2009.

[2] Bayindir, Kamil Çağatay, Mehmet Ali Gözüküçük, and Ahmet Teke. "A comprehensive overview of hybrid electric vehicle: Powertrain configurations, powertrain control techniques and electronic control units", Energy Conversion and Management 52, 2011.

[3] Kumar, Lalit, and Shailendra Jain. "Electric propulsion system for electric vehicular technology: A review", Renewable and Sustainable Energy Reviews 29, 2014.

[4] De Santiago, Juan, et al. "Electrical motor drivelines in commercial all-electric vehicles: a review", IEEE Transactions on Vehicular Technology, 2012.

[5] Melo. P., "A general overview on hybrid and electric vehicles", Neutro à Terra, no 11, 2013.
Numa próxima oportunidade, voltar-se-á a este assunto tão atual quanto em rápida evolução, que, seguramente, justificará um maior aprofundamento e atualização.

[6] "Evolution Electric vehicles in Europe: gearing up for a new phase?", Amsterdam Roundtable Foundation and McKinsey \& Company The Netherlands, 2014.

[7] Agarwal, Vipul, and Mayank Dev. "Introduction to hybrid electric vehicles: State of art ", Engineering and Systems (SCES), 2013 Students Conference on. IEEE, 2013.

[8] Kebriaei, Mohammad, Abolfazl Halvaei Niasar, and Behzad Asaei. "Hybrid electric vehicles: An overview", 2015 International Conference on Connected Vehicles and Expo (ICCVE). IEEE, 2015.

[9] Karden, E. "Microhybrid Goes Mainstream: Battery Selection and Trends", Advanced Automotive Battery Conference Europe. 2011.

[10] "Global EV outlook 2016 - Beyond one million electric cars", International Energy Agency, 2016.

Curiosidade:
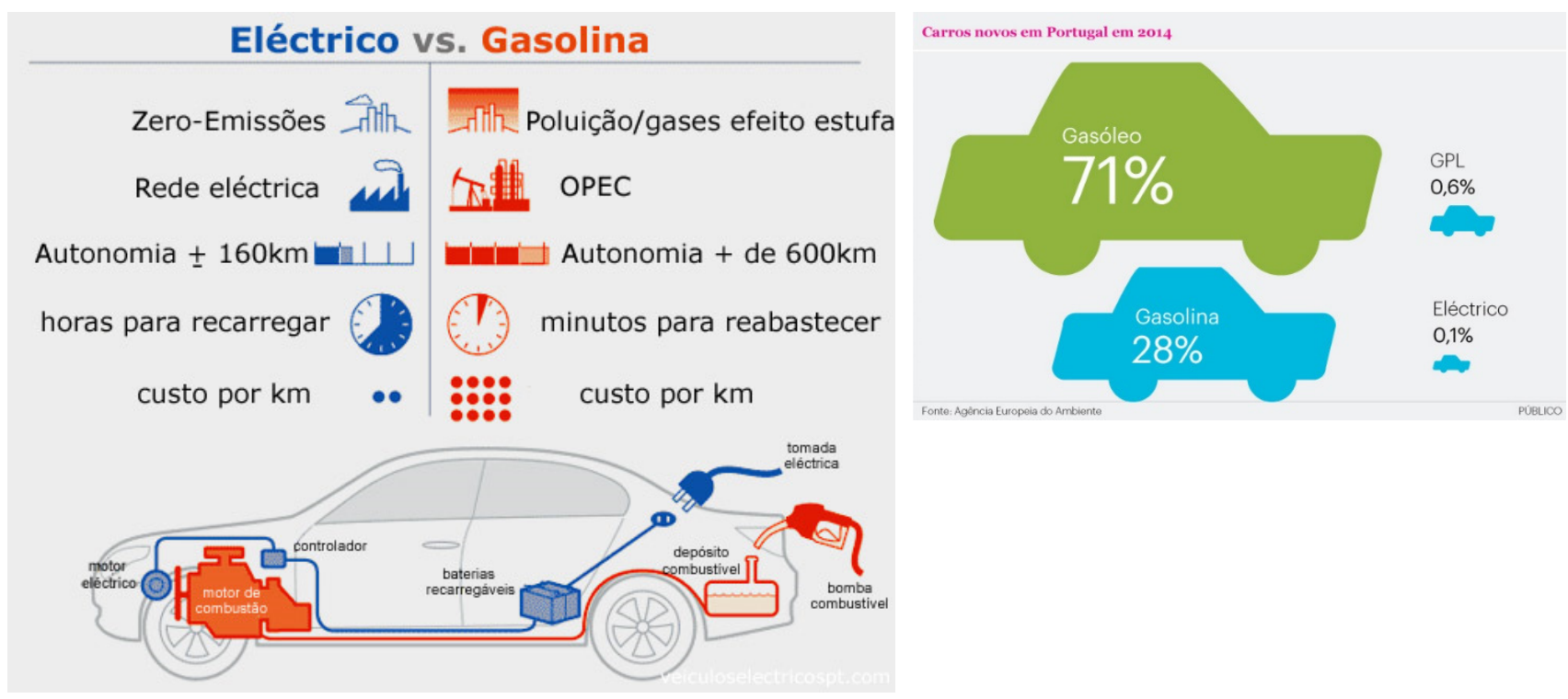